\title{
Parallel-MLFMA Solutions of Large-Scale Problems Involving Composite Objects
}

\author{
Özgür Ergül \\ Department of Mathematics and Statistics \\ University of Strathclyde, Glasgow, UK \\ Email: ozgur.ergul@strath.ac.uk
}

\author{
Levent Gürel ${ }^{1,2}$ \\ ${ }^{1}$ Department of Electrical and Electronics Engineering \\ ${ }^{2}$ Computational Electromagnetics Research Center (BiLCEM) \\ Bilkent University, Bilkent, Ankara, Turkey \\ Email: lgurel@bilkent.edu.tr
}

\begin{abstract}
We present a parallel implementation of the multilevel fast multipole algorithm (MLFMA) for fast and accurate solutions of large-scale electromagnetics problems involving composite objects with dielectric and metallic parts. Problems are formulated with the electric and magnetic current combinedfield integral equation (JMCFIE) and solved iteratively with MLFMA on distributed-memory architectures. Numerical examples involving canonical and complicated objects, such as optical metamaterials, are presented to demonstrate the accuracy and efficiency of the implementation.
\end{abstract}

\section{INTRODUCTION}

In recent years, parallelization of the multilevel fast multipole algorithm (MLFMA) [1] has enabled the solution of extremely large electromagnetics problems discretized with hundreds of millions of unknowns [2]-[6]. On the other hand, most of the previous implementations have been developed for metallic objects and less attention has been paid to dielectric objects, and especially, to more complex structures involving multiple dielectric and metallic parts [2]. Recently, we showed that the hierarchical strategy, which was originally proposed for metallic objects [3],[5], can be applied to homogeneous dielectric objects [6] for efficient parallel simulations. In this work, we further extend the hierarchical strategy to general objects with coexisting multiple dielectric and/or metallic parts. The developed implementation provides fast and accurate solutions of real-life problems, such as metamaterials at optical frequencies, discretized with large numbers of unknowns.

\section{PARAllel ImPlementation}

The parallel implementation consists of the following major components that are summarized briefly.

\section{A. Formulation, Discretization, and Near-Zone Interactions}

Problems are formulated with the electric and magnetic current combined-field integral equation (JMCFIE) and discretized with the oriented Rao-Wilton-Glisson (RWG) functions on small planar triangles. Interactions between nearby basis and testing functions are calculated via singularity extraction and adaptive integration techniques.

\section{B. Iterative Solutions and Far-Zone Interactions}

For each penetrable region and matrix partition (i.e., $\bar{Z}_{J J}$, $\bar{Z}_{J M}, \bar{Z}_{M J}$, and $\bar{Z}_{M M}$ ), a multilevel tree structure is constructed by placing the associated surfaces (interfaces) in a cubic box and recursively dividing them into subboxes. A region may consist of multiple unconnected subregions (with the same electrical parameters), which are considered together in the same tree structure. Hence, an overall matrixvector multiplication (required for iterative solutions) is obtained by tracing different tree structures and superposing interactions in different media. Also note that each trace is a sequence of aggregation, translation, and disaggregation stages, as usually defined for MLFMA. Radiated and incoming fields are sampled with the sampling rate determined by the excess bandwidth formula. Interpolations and anterpolations are carried out using the Lagrange method. Iterative solutions are accelerated by the block-diagonal preconditions.

\section{Parallelization}

Each tree structure (constructed for each medium and partition) is parallelized using the hierarchical strategy, which is based on the simultaneous partitioning of subboxes and field samples at all levels. Specifically, for each tree structure, partitions of subboxes and their samples can be optimized to improve the load balancing and to minimize communications. Considering two-dimensional partitioning [3], three different types of one-to-one communications are required: Vertical communications during aggregation/disaggregation, horizontal communications during translations, and data exchanges between pairs of processes to modify partitioning. Code rearrangements are performed to improve the memory recycling and to enable the solution of large problems on moderate computers.

\section{NumERICAL RESUltS}

Fig. 1 presents the solution of canonical problems involving a spherical object. A dielectric sphere of radius $50 \mu \mathrm{m}$ (core) is placed inside another dielectric sphere of radius $100 \mu \mathrm{m}$ (shell) and located in vacuum (host medium). The relative permittivities of the core and shell are 2.0 and 3.0, respectively. The object is illuminated by a plane wave at $48 \mathrm{THz}, 96 \mathrm{THz}$, and $192 \mathrm{THz}$. At these frequencies, the size of the object corresponds to approximately $16 \lambda_{0}, 32 \lambda_{0}$, and $64 \lambda_{0}$, respectively, where $\lambda_{0}$ is the wavelength in the host medium. For numerical solutions, MLFMA is parallelized into 64 processes using the hierarchical strategy on a cluster of Intel Xeon Nehalem quad-core processors with $2.80 \mathrm{GHz}$ clock rate. The 


\begin{tabular}{|c|c|c|c|}
\cline { 2 - 4 }$\varepsilon_{\mathrm{r}}=2.0$ & Unknowns & Iterations & Total Time \\
\cline { 2 - 4 }$\varepsilon_{\mathrm{r}}=3.0$ & $3,278,208$ & 66 & 1.4 hours \\
\cline { 2 - 4 } & $13,112,832$ & 121 & 9.0 hours \\
\hline $52,451,328$ & 66 & 24 hours \\
\hline
\end{tabular}
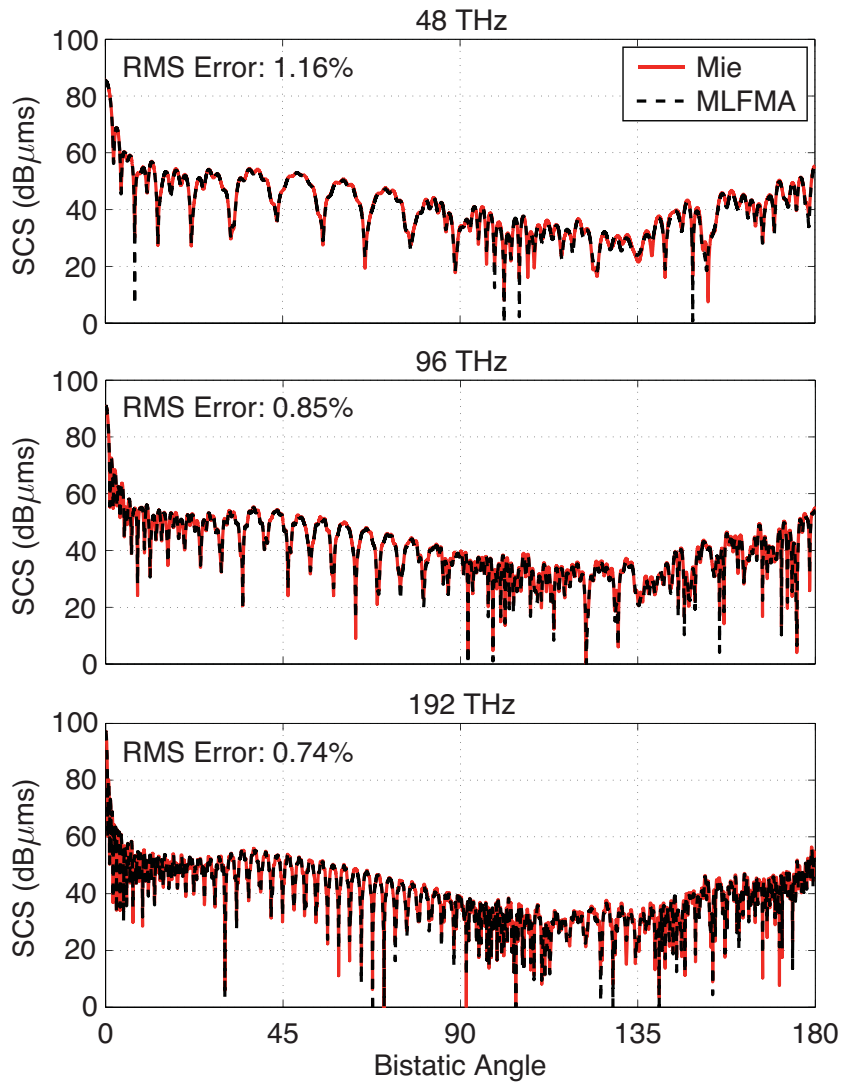

Fig. 1. Solutions of large-scale scattering problems involving a spherical object, which consists of a dielectric core of radius $50 \mu \mathrm{m}$ inside a dielectric shell of radius $100 \mu \mathrm{m}$, at $48 \mathrm{THz}, 96 \mathrm{THz}$, and $192 \mathrm{THz}$.

largest problem (at $192 \mathrm{THz}$ ) is discretized with $52,451,328$ unknowns and solved in approximately 24 hours. Fig. 1 depicts the bistatic scattering cross section (SCS) values (in $\mathrm{dB} \mu \mathrm{ms}$ ) as a function of the bistatic angle from $0^{\circ}$ to $180^{\circ}$, where $0^{\circ}$ corresponds to the forward-scattering direction. Computational values agree well with reference values obtained via analytical Mie-series solutions. The root-mean-square (RMS) error in the RCS values is around $1 \%$ at all three frequencies.

Fig. 2 presents the solution of an electromagnetics problem involving a metamaterial designed for optical frequencies. A total of $101 \times 101=10201$ metallic rods are enclosed in a lossy shell with a complex relative permittivity of $9.6+i 0.8$. Geometries and dimensions of the rods and shell are described in Fig. 2. The structure is illuminated by a plane wave at $417 \mathrm{THz}$. The problem is discretized with $13,224,714$ unknowns and solved by MLFMA (parallelized into 64 processes using the hierarchical strategy on the Intel Xeon cluster) in less than 16 hours. Fig. 2 depicts the total electric field in the vicinity of the structure. Considering only the host medium in
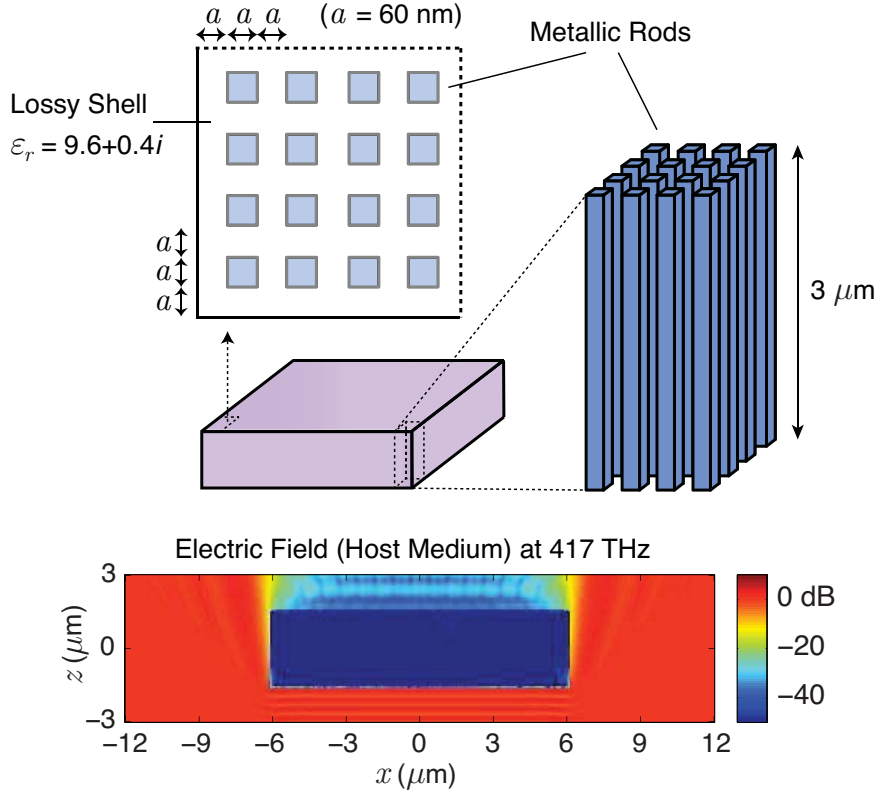

Fig. 2. Solution of an electromagnetics problems involving a metamaterial, which consists of $101 \times 101$ metallic rods inside a lossy shell. The total electric field in the host medium is depicted when the structure is illuminated by a plane wave at $417 \mathrm{THz}$.

accordance with the equivalence principle [6], electromagnetic fields should vanish inside the object. Fig. 2 demonstrates the high accuracy of the solution, since the electric field inside the object is $50 \mathrm{~dB}$ lower than the electric field outside the object.

\section{ACKNOWLEDGMENT}

This work was supported by the Scientific and Technical Research Council of Turkey (TUBITAK) under Research Grants 110E268 and 111E203, by the Engineering and Physical Sciences Research Council (EPSRC) under Research Grant EP/J007471/1, by the Centre for Numerical Algorithms and Intelligent Software (EPSRC-EP/G036136/1), and by contracts from ASELSAN and SSM.

\section{REFERENCES}

[1] W. C. Chew, J.-M. Jin, E. Michielssen, and J. Song, Fast and Efficient Algorithms in Computational Electromagnetics. Boston, MA: Artech House, 2001.

[2] J. Fostier and F. Olyslager, "An asynchronous parallel MLFMA for scattering at multiple dielectric objects," IEEE Trans. Antennas Propag., vol. 56, no. 8, pp. 2346-2355, Aug. 2008.

[3] Ö. Ergül and L. Gürel, "A hierarchical partitioning strategy for an efficient parallelization of the multilevel fast multipole algorithm," IEEE Trans. Antennas Propag., vol. 57, no. 6, pp. 1740-1750, Jun. 2009.

[4] J. M. Taboada, M. G. Araujo, J. M. Bertolo, L. Landesa, F. Obelleiro, and J. L. Rodriguez, "MLFMA-FFT parallel algorithm for the solution of large-scale problems in electromagnetics," Prog. Electromagn. Res., vol. 105, pp. 15-30, 2010.

[5] Ö. Ergül and L. Gürel, "Rigorous solutions of electromagnetics problems involving hundreds of millions of unknowns," IEEE Antennas Propag. Mag., vol. 53, no. 1, pp. 18-26, Feb. 2011.

[6] Ö. Ergül, "Solutions of large-scale electromagnetics problems involving dielectric objects with the parallel multilevel fast multipole algorithm," J. Opt. Soc. Am. A., vol. 28, no. 11, pp. 2261-2268, Nov. 2011. 